\title{
Wound infections after median sternotomy treated by VAC therapy, summary of results, and risk factor analysis
}

\author{
Hulman M, Bezak B, Artemiou P, Cikrai R \\ Clinic of Cardiac Surgery, The National Institute of Cardiovascular Diseases, Bratislava, Slovakia. \\ branislav.bezak@nusch.sk
}

\begin{abstract}
INTRODUCTION: The aim of this study is to summarize results and analyze risk factors for the development of wound infection in heart surgery patients after median sternotomy.

METHOD: In this retrospective analysis with assessment of multiple risk factors, we examined 143 patients with infection after median sternotomy treated with VAC therapy from total of 4,650 patients operated in our department from 2012 to 2015.

RESULTS: Total of 143 patients developed significant SSI treated by VAC therapy following cardiac surgery. Of these, only 14 patients developed DSWI and one patient was diagnosed with suspected osteomyelitis. BMI, female gender, and use of BIMA proved to be statistically significant risk factors in our study $(p<0.001)$. The acuteness of operations did not have a statistically significant effect. However, it had a significant effect on the severity of infection $(p<0.01)$. The severity of infection proved to be a significant prognostic factor for patients' outcome $(p<0.01)$.

CONCLUSION: In our study, BMI, female gender, and use of BIMA (bilateral internal mammary artery) in patients with DM were predictors for the development of SWI. The acuteness of operation did not have a statistically significant effect. However, it had a statistically significant effect on the severity of infection (Tab. 3, Ref. 30). Text in PDF www.elis.sk.

KEY WORDS: wound infections, median sternotomy, VAC therapy, negative-pressure wound closure.
\end{abstract}

\section{Introduction}

Median sternotomy is the most common method of surgical approach in cardiac surgery $(1,2)$. SSI (surgical site infection) in this area remains a much-feared postoperative complication. Despite the progress in prevention, according to literature, this complication still occurs in approximately $3 \%$ of patients (3-5). Some sources however report an incidence of up to $10 \%(6,7)$.

Sternal wound infections are most often divided according to the severity into superficial and deep. Superficial infection (SSWI) affects skin, subcutaneous tissue and pectoral fascia. The incidence of SSWIs ranges from 0.5 to $8 \%$ with an associated morbidity and mortality rate from 0.5 to $9 \%$ (8). Deep sternal infection (DSWI) affects bone, substernal space and mediastinum. This serious complication affects less than $2 \%$ of patients $(9,10)$. On the other hand, in contrast to surface infections, it increases the mortality more than twice (11).

The most commonly reported risk factors for developing this infection include age, female gender, obesity, diabetes mellitus and hyperglycemia, tobacco smoking, chronic obstructive pulmonary disease, surgical technique, use of BIMA (bilateral internal

Address for correspondence: B. Bezák, MD, Clinic of Cardiac Surgery, The National Institute of Cardiovascular Diseases, Pod Krasnou horkou 7185/1, SK-831 01 Bratislava, Slovakia.

Phone: +421.905449075 mammary artery), heart failure, renal failure, peripheral vascular disease, prolonged need for inotropic support, prolonged stay in intensive care, and emergent or urgent surgery (12-15).

There are several methods of treating sternal wound infections. The most commonly used procedures include closed suction antibiotic catheter irrigation systems, vacuum-assisted closure and various flap coverages (8). At our department we experienced the best results with VAC therapy (vacuum-assisted closure) and it is the method of choice for every sternal wound infection not responding to regular dressings. VAC therapy is accepted as a suitable method for the treatment of open infectious wounds. It reduces bacterial colonization of the wound, swelling, exudation, stimulates growth of granulation tissue, improves perfusion and creates favorable microenvironment for wound healing (16-18).

Our study attempts to summarize the results at the Clinic of Cardiac Surgery at The National Institute of Cardiovascular Diseases in Bratislava and analyze several risk factors associated with SSI.

\section{Materials and methods}

\section{Patient selection}

The study was conducted at the Clinic of Cardiac Surgery at The National Institute of Cardiovascular Diseases in Bratislava (NÚSCH). In our retrospective study, we included patients who underwent cardiac surgery at our clinic between years 2012 and 
2015 and developed SSI in the postoperative course treated by VAC therapy. Small uncomplicated infections with ASEPSIS score (19) under 30 that were treated only by daily dressing were not included in the study. Similarly, patients with wound infections at other sites (wound infections after saphenectomy, in inguinal or deltoideopectoral region, etc.) were not included in the study. We analyzed the risk factors of 143 patients who developed SSI after median sternotomy and were treated with VAC therapy from total of 4,650 operated patients.

\section{Statistical analysis}

After processing individual data, we used Fisher's exact test to determine statistical significance. We preferred Fisher's test over X2-test because some of the analyzed groups of patients were relatively small and would invalidate the X2-test. The probability randomness threshold was set at 0.01 , meaning there is substantial evidence against the null hypothesis. Numerical variables were presented as mean with minimal, maximal and median values, and categorized variables were summarized by percentages. For the statistical analysis we used statistical analysis tools available at http://www.socscistatistics.com.

An informed consent and institutional review board permission were obtained to present these results.

\section{Results}

\section{Incidence}

In the period from 2012 to 2015, a total of 143 patients (3.08\%) developed significant SSI treated by VAC therapy following cardiac surgery. Of these, only 14 patients developed DSWI (0.3 \% of total patients) and one patient was diagnosed with suspected osteomyelitis.

The study population consisted of a total of 74 men aged 38-83 years and 69 women aged 29-85 years. The mean age of men and women was 63 and 67 years, respectively. The most common primary diagnoses among SSI group were coronary heart disease (64\%) and aortic stenosis (8\%). The most common operations were CABG (69 \%) and aortic valve replacement (8\%). We analyzed

\section{Tab. 1. Infectious agents cultivated from wound swabs.}

\begin{tabular}{lc}
\hline Infectious agent & Percentage of infected wounds \\
\hline Staphylococcus epidermidis & $25.17 \%$ \\
Enterococcus faecalis & $22.38 \%$ \\
Unknown & $17.48 \%$ \\
Staphylococcus aureus & $17.48 \%$ \\
Pseudomonas aureginosa & $7.69 \%$ \\
Escherichia coli & $6.99 \%$ \\
MRSA & $6.99 \%$ \\
Klebsiella pneumoniae & $5.59 \%$ \\
Proteus mirabilis & $4.9 \%$ \\
Klebsiella oxytoca & $4.2 \%$ \\
Staphylococcus haemolyticus & $3.5 \%$ \\
Negative & $2.8 \%$ \\
Staphylococcus hominis & $2.8 \%$ \\
Staphylococcus lugdunensis & $2.8 \%$ \\
Fungi & $2.8 \%$ \\
Other & $18.88 \%$ \\
\hline
\end{tabular}

Tab. 2. Impact of acuteness of operation on the severity of SWI $(\mathrm{p}<0.01)$.

\begin{tabular}{lccc}
\hline $\begin{array}{l}\text { Acuteness } \\
\text { of operation }\end{array}$ & $\begin{array}{c}\text { Number } \\
\text { of patients }\end{array}$ & $\begin{array}{c}\text { Superficial } \\
\text { wounds }\end{array}$ & $\begin{array}{c}\text { Deep } \\
\text { wounds }\end{array}$ \\
\hline Planned & 129 & 121 & 8 \\
Emergent & 9 & 4 & 5 \\
Urgent & 5 & 4 & 1 \\
\hline
\end{tabular}

5 risk factors in association with VAC therapy, including female gender, obesity, diabetes mellitus and hyperglycemia, use of BIMA (bilateral internal mammary artery), and emergency of surgery.

A majority of patients was suitable for secondary suture of the wound after the infection had been treated (two sterile cultivation results from wound swabs and growth of granulation tissue). Part of the patients with extensive damage however, required reconstructive surgery using tissue flaps. Of the total number of wounds, superficial were present in $85 \%$, deep in $10 \%$, complicated superficial infections in $4 \%$ and suspected osteomyelitis in $1 \%$ of patients. The most common pathogens isolated from wound swabs were Staphylococcus epidermidis (25.17\%), Enterococcus faecalis (22.38 \%) and Staphylococcus aureus (17.48\%). Other relatively frequent agents were Pseudomonas aureginosa (7.69 \%), MRSA (6.99\%) and E. coli (6.99\%). These data are presented in Table 1.

\section{$B M I$}

In the SSI group, the average BMI was higher (30.72; min: 18.8; max: 45.062; median: 30.67) compared to the average BMI of all operated patients (28.48). In the SSI group, only $13 \%$ of patients had normal BMI; $31 \%$ of patients were overweight, 51 $\%$ obese and $6 \%$ morbidly obese (BMI $>40)$.

\section{Gender}

The SSI group had a significantly higher proportion of female patients compared to the group of all operated patients (48.25\% and $31.38 \%$, respectively). In our study, the female gender was confirmed to be a statistically significant factor ( $p<0.01$ ).

\section{Diabetes mellitus and use of two arterial grafts in $C A B G$}

The use of bilateral internal mammary artery for myocardial revascularization in patients with diabetes mellitus proved to be a significant risk factor for developing SSI ( $\mathrm{p}<0.01)$. Patients with this combination of risk factors had a 2.5 times higher risk of developing SSI compared to total risk (7.79\% and $3.08 \%$, respectively).

Data summarizing risk factors, namely BMI, gender and DM + BIMA are presented in Table 2.

\section{Severity of infection}

Development of DSWI significantly worsens the prognosis of patient compared to SSWI. In the group of 14 patients with DSWI. 9 (64 \%) patients died, 4 (29\%) were discharged from hospital or transferred to other departments, and 1 patient (7\%) had extensive tissue damage requiring tissue flap surgery. In the group with SSWI, $84 \%$ patients were discharged, the mortality was $11 \%$, and $5 \%$ of patients required tissue flap surgery. The severity of infection is thus a statistically significant prognostic factor $(\mathrm{p}<0.01)$. 
Tab. 3. Risk factors.

\begin{tabular}{lccc}
\hline Risk factor & All patients & SWI group & p \\
\hline Average BMI [kg.m ${ }^{2}$ ] & 28.48 & 30.72 & $<0.01$ \\
Proportion of female patients & $31.38 \%$ & $48.25 \%$ & $<0.01$ \\
Diabetes mellitus + use of BIMA & $3.08 \%$ & $8 \%$ & $<0.01$ \\
\hline
\end{tabular}

\section{Acuteness of the operation}

The acuteness of operations did not have a statistically significant effect on the development of SSI. However, it had a statistically significant effect on the severity of infection $(\mathrm{p}<0.01)$. In the group of electively operated patients with SSI, only $6.2 \%$ developed DSWI. In contrast, patients in urgent group developed DSWI in $20 \%$ while in emergent group in as much as $56 \%$. These data are presented in Table 3.

\section{Discussion}

In the period between 2012 and 2015, $3.08 \%$ of patients developed SSI after median sternotomy. The incidence is slightly lower than the actual incidence since we included only patients treated with VAC therapy. The common practice at our department is to use VAC therapy for every infection not manageable by standard dressing. These results still compare to the incidence spectrum of SSI most commonly reported in literature, which is approximately $3 \%$ of patients (3-4). Some sources however report the incidence of up to $10 \%(6,7)$.

The incidence of DSWI in our study was $0.3 \%$ and every patient with DSWI was treated by deep VAC therapy. Compared to other studies, this incidence is on the lower end of the spectrum $(9,10,20)$. The mortality associated with DSWI in our study was however significantly higher (64\%) compared to the results of other studies which range from 10 up to $40 \%$ (18). These results may be biased due to the small number of patients in the DSWI group (14 patients over the period of 4 years) but require a further investigation nonetheless.

BMI is a widely acknowledged risk factor for the development of SSI (12-15). According to a study from Brazil (21), the odds ratio for developing SWI in patients with BMI > 30 is 1.56 while in patients with BMI $>40$, it is 6.27. The average BMI in the SSI group in our study was 30.72 . Only $13 \%$ of patients had normal BMI; $31 \%$ of patients were overweight, $51 \%$ obese and $6 \%$ morbidly obese (BMI $>40$ ).

The infectious agents associated with SSI were most commonly skin commensals (Staphylococcus epidermidis; $25.17 \%$ ) and microorganisms originating in the digestive tract (Enterococcus faecalis; $22.38 \%$ ). These results correlate with the previous study by Lamaignen et al (22).

In our study, the female gender was confirmed to be a statistically significant factor $(\mathrm{p}<0.01)$. The SSI group had a significantly higher proportion of female patients compared to the group of all operated patients (48.25\% and $31.38 \%$, respectively). A study from Tehran Heart Center reported results very comparable to ours (52.3 \% compared to $25.5 \%$ ) (23). This study interestingly also states that female gender is not viewed as a significant risk factor by other related investigations.
In our experience, one of the most feared combinations of risk factors is diabetes mellitus and the use of two arterial grafts in CABG. Arterial grafts are superior to venous ones in terms of patency (24). Use of two arterial grafts (BIMA, bilateral use of IMA - left and right internal mammary artery) for myocardial revascularization is therefore a favorable alternative compared with the use of one arterial graft and one venous graft $(24,25)$. Although there are still no conclusive data, the use of BIMA improves survival and reduces the need for redo surgery (26). This procedure however carries an increased risk of developing SSI due to the disruption of blood supply to this area. In our study, patients with this combination of risk factors had a 2.5 times higher risk of developing SSI compared to total risk (7.79 \% and $3.08 \%$, respectively). In contrast, a more recent study (27) states that the use of BIMA was not associated with a significantly higher morbidity.

The acuteness of operations in our study did not have a statistically significant effect on the development of SSI. However, it had a statistically significant effect on the severity of infection. Other studies $(28,29)$ also report higher risk of developing DSWI in emergent or urgent operations. But according to an older study (30), the emergency of operation affects the incidence of SSI in general, and not only the severity of infection as we observed.

There are several limitations to our study. It is a single-center retrospective study. The investigation of patients was limited to the hospital admission and did not include long-term follow-up after discharge. The fact that the number of patients in DSWI group was relatively small could cause a bias.

In conclusion VAC therapy is an effective treatment for the management of SWI. Our results are comparable with the results reported in literature. BMI, female gender, and use of BIMA are predictors for the development of SWI in our study.

\section{References}

1. Dürrleman N, Massard G. Modified lateral thoracotomy. Multimedia Manual of Cardio-Thoracic Surgery 2006; 0810 (2006): mmcts-2006.

2. Oda, T. Operative technique of median sternotomy. Kyobu geka. Jap J Thorac Surg 2010; 63 (Suppl 8): 632-635.

3. Lepelletier D et al. Surgical-site infection after cardiac surgery: incidence, microbiology, and risk factors. Infect Control Hosp Epidemiol 2005; 26 (5): 466-472.

4. Marković-Denić $\mathbf{L}$ et al. Incidence of surgical site infection after open heart surgery. Acta Chir Iugosl 2009; 57 (2): 45-48.

5. Si D et al. Surgical site infections following coronary artery bypass graft procedures: 10 years of surveillance data. BMC Infect Dis 2014; 14 (1): 1.

6. Finkelstein R et al. Surgical site infection rates following cardiac surgery: the impact of a 6-year infection control program. Amer J Infect Control 2005; 33 (8): 450-454.

7. Cove ME, Spelman DW, MacLaren G. Infectious complications of cardiac surgery: a clinical review. J Cardiothor Vasc Anesth 2012; 26 (6): 1094.

8. Kimberly S, Anderson E, Harper JG. Overview and management of sternal wound infection. Semin Plast Surg 2011; 25 (1).

9. Hiroshi K et al. Deep sternal wound infection after cardiac surgery. J Cardiothor Surg 2013; (8): 1. 
10. Filsoufi $\mathbf{F}$ et al. Epidemiology of deep sternal wound infection in cardiac surgery. J Cardiothor Vasc Anesth 2009; 23 (4): 488-494.

11. Braxton $\mathbf{M}$ et al. 10-year follow-up of patients with and without mediastinitis. Semin Thor Cardiovasc Surg 2004; 16 (1): 70-76.

12. Omran AS et al. Superficial and deep sternal wound infection after more than 9000 coronary artery bypass graft (CABG): incidence, risk factors and mortality. BMC Infect Dis 2007; 7 (1): 1.

13. Group The Parisian Mediastinitis Study. Risk factors for deep sternal wound infection after sternotomy: a prospective, multicenter study. J Thor Cardiovasc Surg 1996; 111 (6): 1200-1207.

14. Cotogni P, Barbero C, Rinaldi M. Deep sternal wound infection after cardiac surgery: Evidences and controversies. World J Crit Care Med 2015; 4 (4: 265.

15. Borger MA et al. Deep sternal wound infection: risk factors and outcomes. Ann Thorac Surg 1998; 65 (4): 1050-1056.

16. Yoshimoto A et al. Efficacy of vacuum-assisted closure therapy on rehabilitation during the treatment for surgical site infection after cardiovascular surgery. Gen Thorac Cardiovasc Surg 2016; 1-6.

17. Simek $\mathbf{M}$ et al. Vacuum-assisted closure in the treatment of sternal wound infection after cardiac surgery. Biomed Pap 2007; 151 (2): 295-299.

18. Fleck TM et al. The vacuum-assisted closure system for the treatment of deep sternal wound infections after cardiac surgery. Ann Thorac Surg 2002; 74 (5): 1596-1600.

19. Ashby $\mathbf{E}$ et al. How will surgical site infection be measured to ensure high quality care for all”? Bone Joint J 2010; 92 (9: 1294-1299.

20. Priyadharshanan A, Bland M, Loubani M. Risk factors and mortality associated with deep sternal wound infections following coronary bypass surgery with or without concomitant procedures in a UK population: a basis for a new risk model? Interact Cardiovasc Thorac Surg 2010; 11 (5): 543-546.
21. Farsky PS et al. Risk factors for sternal wound infections and application of the STS score in coronary artery bypass graft surgery. Brazil J Cardiovasc Surg 2011; 26 (4): 624-629.

22. Lemaignen A et al. Sternal wound infection after cardiac surgery: incidence and risk factors according to clinical presentation. Clin Microbiol Infect 2015; 21 (7): 674-e11.

23. Omran AS et al. Superficial and deep sternal wound infection after more than 9000 coronary artery bypass graft (CABG): incidence, risk factors and mortality. BMC Infect Dis 2007; 7 (1): 112.

24. Taggart DP. Current status of arterial grafts for coronary artery bypass grafting. Ann Cardiothor Surg 2013; 2 (4): 427-430.

25. Vallely MP, James J, Edelman B, Wilson MK. Bilateral internal mammary arteries: evidence and technical considerations. Ann Cardiothorac Surg 2013; 2 (4): 570.

26. Taggart DP. Bilateral internal mammary artery grafting: are BIMA better? (2002): 7-9.

27. Lenz K et al. Coronary artery bypass surgery in diabetic patients-risk factors for sternal wound infections. GMS Interdiscipl Plast Reconstr Surg DGPW 2016; 5.

28. Sakamoto $\mathbf{H}$ et al. Risk factors and treatment of deep sternal wound infection after cardiac operation. Ann Thorac Cardiovasc Surg 2003; 9 (4): 226-232.

29. Chan M et al. A retrospective study of deep sternal wound infections: clinical and microbiological characteristics, treatment, and risk factors for complications. Diagn Microbiol Infect Dis 2016; 84 (3): 261-265.

30. Sofer $D$ et al. Sternal wound infections in patients after coronary artery bypass grafting using bilateral skeletonized internal mammary arteries. Ann Surg 1999; 229 (4): 585.

Received April 26, 2017. Accepted June 27, 2017. 